\title{
Effect of electric field on defect generation and migration in $\mathrm{HfO}_{2}$
}

\author{
Jack W. Strand $\odot$ \\ Università di Modena e Reggio Emilia, Via Amendola 2, 42122 Reggio Emilia, Italy \\ and Department of Physics and Astronomy, University College London, Gower Street, London WC1E 6BT, United Kingdom \\ Jonathon Cottom $\odot$ \\ Department of Physics and Astronomy, University College London, Gower Street, London WC1E 6BT, United Kingdom \\ Luca Larcher \\ Applied Materials, NMS, Via Sicilia 31, 42122 Reggio Emilia, Italy \\ Alexander L. Shluger \\ Department of Physics and Astronomy, University College London, Gower Street, London WC1E 6BT, United Kingdom
}

(Received 20 April 2020; accepted 17 June 2020; published 21 July 2020)

\begin{abstract}
Understanding the effect of electric fields on defect creation and diffusion in metal oxides is of fundamental importance for developing accurate models of oxide degradation in electronic devices and dielectric breakdown. We use the Berry phase operator method within density functional theory to calculate how an applied electric field affects barriers for the creation of oxygen vacancy-interstitial defect pairs (DPs) and diffusion of interstitial $\mathrm{O}$ ions in monoclinic $(m-) \mathrm{HfO}_{2}$. The results demonstrate that even close to breakdown fields, barriers for DP generation exceed $6 \mathrm{eV}$ in the perfect $m-\mathrm{HfO}_{2}$ lattice. Simulated injection of extra electrons from electrodes significantly lowers barriers for the creation of DPs, which are further reduced by the field to around $1 \mathrm{eV}$. Thus, bias application facilitates the injection of electrons into the oxide; these extra electrons reduce energy barriers for the creation of $\mathrm{O}$ vacancies, and these barriers as well as those for $\mathrm{O}$ ion diffusion are further lowered by the field. We find that, within a linear regime, the electric field modulates the barrier height by a dot product between the electric field and the electric dipole at the zero-field transition state to good accuracy.
\end{abstract}

DOI: 10.1103/PhysRevB.102.014106

\section{INTRODUCTION}

It has been well established that degradation of dielectric properties and time-dependent dielectric breakdown of gate oxides, such as $\mathrm{SiO}_{2}$ and $\mathrm{HfO}_{2}$, under bias stress are directly related to defect generation processes in the oxide layer [1-4]. More recently, these processes were reviewed in, e.g., [5,6]; however, the nature of defects and the mechanisms of their creation are still poorly understood. Strong evidence exists that electron and hole injection as well as hydrogen interdiffusion from the top electrode can be involved in the degradation mechanism [5]. Some other models (e.g., the thermochemical $E$ model, where $E$ is the electric field strength $[7,8]$ ) are solely based on Me-O bond breaking and defect creation in oxides as a result of electric field application and polarization of the dielectric and do not involve extra carriers and/or hydrogen.

On the other hand, the so-called $1 / E$ model [9] assumes that degradation happens as a consequence of FowlerNordheim injection of electrons from the substrate into the oxide conduction band; these electrons are accelerated by the applied $\mathbf{E}$ field to the anode where damage occurs as a result of impact ionization. Further, in the case of $\mathrm{SiO}_{2}$, holes injected from the anode are expected to be trapped onto weakened bonds. In this model, the time to breakdown is predicted to follow an exponential dependence on the reciprocal of the electric field $1 / E$. Although hole injection into $\mathrm{SiO}_{2}$ is believed to be inefficient due to the large band offset, experimental evidence suggests that hole injection into $\mathrm{HfO}_{2}$ leads to degradation [10,11].

In all these models the dominant causes of defect creation and ensuing breakdown are the electric field $\mathbf{E}$, injected holes, and electrons, respectively. The so called dominant carrier change model attempts to reconcile these models by suggesting that both electrons and holes contribute to the defect generation to induce breakdown and that which carrier dominates depends on the conditions, such as the stress voltage [12]. However, the exact role of carriers injected into the oxide as a result of the field application and the interaction of these carriers with the field is not easy to fully ascertain. The model proposed in [13-15] attempts to provide such a link. It suggests a mechanism related to how electrons and holes injected into oxide film can facilitate the creation of $\mathrm{O}$ vacancies and that the energy barrier for this process is, in turn, also lowered by the field. The field also affects the rates of electron transfer through $\mathrm{O}$ vacancies and diffusion of $\mathrm{O}$ vacancies and interstitial ions.

The ability of this model to describe the time-dependent dielectric breakdown (TDDB) in amorphous $\mathrm{SiO}_{2}$ films was examined in Ref. [16] using numerical simulations implemented in the GINESTRA package [17]. These simulations account for 
the fact that electric field reduces barriers for defect generation and ion diffusion in dielectrics using an approximation where the barrier reduction for vacancy generation is proportional to the strength of the field [16]. The same approximation has long been used to describe ion mobility in electric field in solids (see, e.g., $[18,19]$ ) and has been applied to describe breaking of Me-O bonds in oxide films in the thermochemical model [8]. In particular, time to breakdown in this model is written as

$$
T_{\mathrm{TDDB}}=\tau_{0} \exp \left(\frac{E_{\mathrm{B}}-\gamma E}{k_{B} T}\right),
$$

where $T_{\mathrm{TDDB}}$ is the time to dielectric breakdown, $E_{\mathrm{B}}$ is the activation energy (energy barrier) for Me-O bond breaking (defect formation), and $E$ is the electric field strength. The key parameters for the model are $E_{\mathrm{B}}$ and $\gamma$, where $\gamma$ is understood as the "field acceleration parameter." It is expressed as [7]

$$
\gamma=p_{0}\left(\frac{2+\kappa}{3}\right),
$$

where $\kappa$ is the dielectric constant and $p_{0}$ is the molecular dipole moment (of the bond to be broken) component in the direction opposite the applied electric field. However, effective dipole moments used in the literature are often extracted from approximate models of defect properties [8]. Moreover, fields responsible for the degradation and dielectric breakdown of oxide films often reach several megavolts per centimeter, where the linear approximation may break.

Our ultimate aim is to test the ability of the electron and hole injection models [13-15] to describe the TDDB in crystalline and amorphous $\mathrm{HfO}_{2}$ using the multiscale methodology of the type implemented in the GINESTRA package. Previous simulations [20] used Eqs. (1) and (2) with empirical parameters to describe defect formation and the TDDB in polycrystalline $\mathrm{HfO}_{2}$ films. We would like to investigate whether it is possible to accurately predict the macroscopic degradation parameters, i.e., the TDDB statistics and its voltage dependence, using the defect characteristics derived from density functional theory (DFT) simulations. In this paper we focus on investigating the effect of the $\mathbf{E}$ field on the barriers for defect creation and diffusion in monoclinic $(m)$ $\mathrm{HfO}_{2}$ using DFT simulations to provide reliable parameters for TDDB simulations to be described in detail in a separate study.

Efficient numerical implementations of the Berry phase operator approach within the modern theory of polarization [21-25] opened new opportunities for testing the assumptions and approximations common to the models of oxide degradation and dielectric breakdown. Recently, we studied the effect of the $\mathbf{E}$ field on diffusion barriers of oxygen vacancies and interstitial oxygen ions in the bulk of $\mathrm{MgO}$ using the Berry phase operator implementation in CP2K [26]. We showed how the dipole moment of the system in different charge states changes as defects migrate. The dipole moment change along the migration pathway can be broken down into two components, one being the change in polarization as the electronic structure changes along the migration pathway, while the second comes from the migration of the net excess charge as such. The effective dipole moment $\mu_{\text {eff }}$ determines how the applied external field affects the migration barrier.
Naturally, it depends on defect polarizability and changes much stronger for charged $\mathrm{O}$ interstitials rather than for $\mathrm{O}$ vacancies [26].

Here we apply the Berry phase operator method to investigate the effects of the applied electric field on the barriers for creation of $\mathrm{O}$ vacancies $V_{\mathrm{O}}$ and interstitial $\mathrm{O}$ ions $\mathrm{O}_{\mathrm{I}}$ and diffusion of $\mathrm{O}_{\mathrm{I}}$ in monoclinic $\mathrm{HfO}_{2}$ in the context of the model of defect creation in $\mathrm{HfO}_{2}$ facilitated by electron injection. This model suggests that the creation of $\left(\mathrm{V}_{O}+\mathrm{O}_{\mathrm{I}}\right)$ defect pairs in perfect $m$ - [14] or amorphous $(a)-\mathrm{HfO}_{2}$ [15] requires overcoming the energy barrier of about $6.0 \mathrm{eV}$. However, the electron injection and trapping in polaronlike states at intrinsic precursor sites or at $\mathrm{O}$ vacancies significantly lowers this barrier to below $2 \mathrm{eV}$ [14]. Such a barrier reduction is achieved after trapping two electrons in a so-called bipolaron state or by an $\mathrm{O}$ vacancy [14]. Our previous calculations demonstrated that $\mathrm{O}_{\mathrm{I}}$ diffusion barriers in $\mathrm{HfO}_{2}$ depend on the charge state of $\mathrm{O}_{\mathrm{I}}$ and are typically below $0.5 \mathrm{eV}$ [14].

We demonstrate that the electric field up to $10 \mathrm{MV} / \mathrm{cm}$ significantly reduces the activation barrier for $\left(V_{\mathrm{O}}+\mathrm{O}_{\mathrm{I}}\right)$ pair creation and $\mathrm{O}_{\mathrm{I}}$ diffusion and provide realistic values for effective dipole moment changes associated with these processes. Our results support the model of defect generation facilitated by electrons (and hole injection) and can be used for simulation of dielectric breakdown in real devices using GINESTRA [17] and other similar methodology [27].

\section{METHODOLOGY}

All calculations are performed using the Gaussian and plane waves [28] method as implemented in the $\mathrm{CP} 2 \mathrm{~K}$ code [29]. The PBE0-TC-LRC (PBE0 with "truncated Coulomb" and "Long Range Correction") [30] non-local functional was selected to accurately reproduce the band gap of $m-\mathrm{HfO}_{2}$ and used in conjunction with the auxiliary density matrix method [31] to mitigate the computational expense. This particular implementation of the hybrid Perdew-BurkeErnzerhof (PBE0) functional abruptly truncates the exactexchange part of the exchange correlation functional above some value of the truncation radius $R_{c}$. Above this range separation, the truncated exchange is replaced by an approximation of the exchange using a generalized gradient approximation PBE exchange-hole formalism [30]. Finally, we used the molecularly optimised double- $\zeta$ basis set with polarisation basis functions from Ref. [32] along with the corresponding pseudopotentials of Goedecker, Teter and Hutter [33] and a 400-Ry plane wave cutoff.

The calculations were performed using 96- and 324-atom supercells in the $\Gamma$ point of the Brillouin zone. Calculations in 96- and 324-atom cells predict band gaps of 6.5 and $6.7 \mathrm{eV}$, respectively, for $m-\mathrm{HfO}_{2}$. The 96-atom supercells are used for interstitial oxygen calculations, whereas 32-atom cells are used for defect pair (DP) calculations owing to greater lattice distortion. We note that $m-\mathrm{HfO}_{2}$ has a quasilayered structure, with alternating planes of three-coordinated (3C) and four-coordinated (4C) O ions shown in Fig. 1. The effect this has on hole polaron trapping was discussed in Ref. [34]. Nascent DPs in $\mathrm{HfO}_{2}$ also form in one of the $\mathrm{O}$ sublattices and can propagate in multiple directions, for example, either 

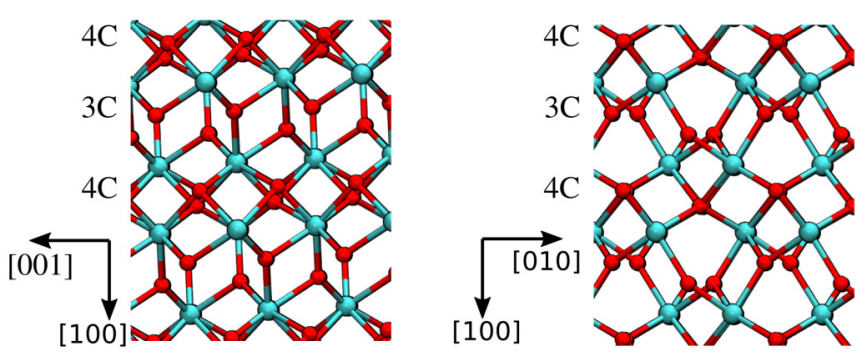

FIG. 1. Two projections of the $m-\mathrm{HfO}_{2}$ structure. Planes of threeand four-coordinated $\mathrm{O}$ ions are indicated.

through the $3 \mathrm{C} \mathrm{O}$ sublattice (in the [001] direction) or through the $3 \mathrm{C}$ and $4 \mathrm{C} \mathrm{O}$ planes in the [100] direction.

The effect of the $\mathbf{E}$ field is included using the Berry phase operator theory within periodic boundary conditions [21-25]. In this approach, polarization is calculated as a Berry phase of the Kohn-Sham (KS) Bloch states. The electric enthalpy functional can be written as

$$
H_{E}[n(\mathbf{r}) ; \mathbf{E}]=E_{\mathrm{KS}}[n(\mathbf{r}) ; \mathbf{E}]-\Omega \mathbf{P}[n(\mathbf{r}) ; \mathbf{E}] \cdot \mathbf{E},
$$

where $n(\mathbf{r})$ is the electron density, $\Omega$ is the cell volume, and $\mathbf{P}$ is the polarization vector of the periodic cell. Polarization in periodic systems is not, in fact, well defined. Values of polarization (of the periodic cell) belong to a "polarization lattice," wherein polarization values separated by one polarization lattice vector are equivalent (see, e.g., [25]). However, differences (that is, changes) in polarization are well defined, provided there are no jumps between branches of the polarization lattice [26]. In all results presented in this work, calculated dipole moments have been analyzed to ensure such jumps have not happened.

DFT calculations carried out using the Berry phase method are self-consistent and take into account changes in the electron density induced by the electric field. However, they are impractical in the context of multiscale simulations of the type used in the GINESTRA platform, which involve many types of defects and different biases. In these simulations one must resort to simpler approximations, such as Eq. (1). At moderate field strengths, where the electron density does not change significantly upon application of the field, one can estimate how the barrier energy has changed due to an applied field as [26]

$$
\Delta E_{B}^{F}=-\left(\vec{\mu}_{2}-\vec{\mu}_{1}\right) \cdot \mathbf{E}=-\vec{\mu}_{\text {eff }} \cdot \mathbf{E} .
$$

In this approximation, the effective dipole moment of the transition state at zero field determines the extent of the barrier change in a field. We will use an effective dipole moment $\vec{\mu}_{\text {eff }}$ at zero field together with Eq. (4) to compare the barrier changes induced by the field calculated using this approximation with those calculated using the Berry phase method. If accurate enough, this approximation should allow one to estimate reaction barrier changes using the change of the dipole moment in zero field instead of considering the full polarization of the system at a given field strength.

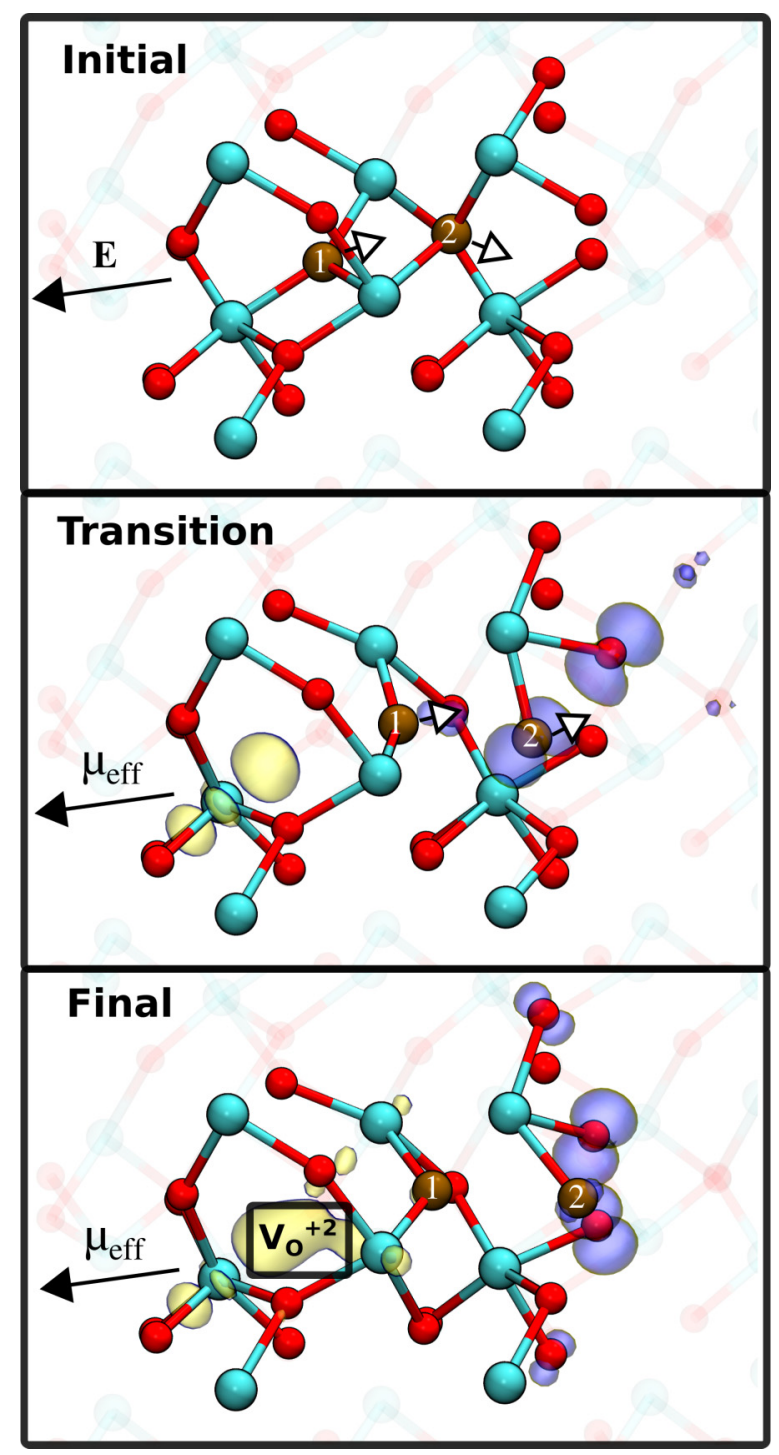

FIG. 2. A fragment of the perfect $m-\mathrm{HfO}_{2}$ lattice where the O DP generation mechanism takes place. Shown are the initial, transition, and final states of the process. Hf ions are light blue, and $\mathrm{O}$ ions are red. The defects are separated via an interstitialcy mechanism, where two oxygen ions collectively move to produce a vacancy-interstitial pair bridged by an oxygen ion. The two $\mathrm{O}$ ions which move, labeled 1 and 2, are distinguished in a dark brown color. In the final state, a $V_{\mathrm{O}}^{+2}+\mathrm{O}_{\mathrm{I}}^{-2}$ pair is created. Yellow surfaces show the vacancy states, and blue surfaces show the $2 p \mathrm{O}$ ion states (isovalue $=0.007$ ).

\section{RESULTS}

\section{A. Formation of a neutral defect pair}

Most degradation models assume the formation of $\mathrm{O}$ vacancies in $\mathrm{HfO}_{2}$ under bias. Such vacancies can exist in $\mathrm{HfO}_{2}$ in five different charge states, depending on the Fermi level position $[35,36]$. Hf-O bond breaking in perfect $m$ - $\mathrm{HfO}_{2}$ can create $\mathrm{O}$ vacancies and $\mathrm{O}$ interstitials in different charge states $[13,37]$. The lowest formation energy corresponds to the creation of a $\left(V^{+2}+\mathrm{O}_{\mathrm{I}}^{-2}\right)$ DP (see Fig. 2). The defect separation process happens via the so-called interstitialcy mechanism characteristic for $\mathrm{O}$ diffusion in oxides [38]. A three-coordinated $\mathrm{O}$ ion (oxygen 1 in Fig. 2) moves along 


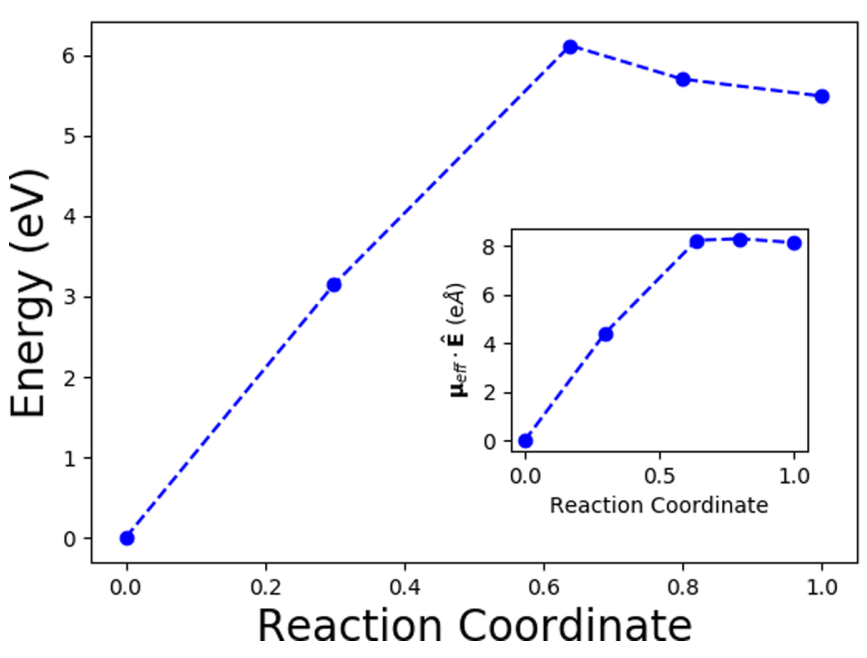

FIG. 3. Energy profile for the generation of a $V_{\mathrm{O}}^{+2}+\mathrm{O}_{\mathrm{I}}^{-2}$ pair in $m-\mathrm{HfO}_{2}$. The barrier height is above $6 \mathrm{eV}$. The inset shows the evolution of the induced dipole, with the $y$ axis corresponding to the induced dipole projected onto the dipole vector at the transition state.

the [100] axis (see Fig. 1) into a 4C O site, displacing the 4C $\mathrm{O}$ ion (oxygen 2 in Fig. 2) farther along the [100] axis into an interstitial position in the $3 \mathrm{C} \mathrm{O}$ sublattice. In this configuration $\mathrm{O}_{\mathrm{I}}^{-2}$ is effectively shielded by a $4 \mathrm{C} \mathrm{O}$ ion from recombining with the $V^{+2}$ vacancy. One can see that the $\mathrm{O}$ ions form an antibonding state. The effective separation of $\mathrm{O}_{\mathrm{I}}^{-2}$ from the vacancy is about $5.5 \AA$; however, the displacement of the individual ions involved in the process is approximately only half this distance due to their concerted motion.

The energy profile for creation of such a DP with respect to the displacement of the pair's center of mass, obtained using the nudged elastic band method [39-41], is shown in Fig. 3. The energy barrier to DP formation is about $6.1 \mathrm{eV}$. The transition state is shown in Fig. 2. We note that the creation of a close $V_{\mathrm{O}}^{+2}+\mathrm{O}_{\mathrm{I}}^{-2}$ pair is not possible along other crystal directions. For example, DPs which exist purely within the 3C sublattice (see Fig. 1) and have a defect axis along the [001] crystal direction will recombine for separations less than 7.0 A. The "reaction coordinate" in Fig. 3 is defined for the $n$th replica as $Q_{n}=\frac{\sum_{i}^{n} d_{i}}{\sum_{i}^{N} d_{i}}$, where $d_{i}$ is the displacement magnitude between the $i$ and $i-1$ replicas. $Q_{0}$ is set as zero, and $N$ is the total number of replicas. This coordinate thus represents the progression of the reaction, with 1.0 indicating that the final state has been reached.

The electric field alignment in all further calculations is chosen to cause the greatest possible reduction to the energy barrier height. This is achieved by setting the $\mathbf{E}$ field parallel to the induced dipole vector at the transition state. In the case of the pathway shown in Fig. 2, the electric field direction is very nearly parallel to the [100] crystal direction. The evolution of the magnitude of the induced dipole along the reaction coordinate of the $\left(V_{\mathrm{O}}^{+2}+\mathrm{O}_{\mathrm{I}}^{-2}\right)$ defect pair formation is shown in the inset of Fig. 3. The application of an electric field with a strength of $5 \mathrm{MV} / \mathrm{cm}$ reduces the barrier to about $6.2 \mathrm{eV}$, and a field of $10 \mathrm{MV} / \mathrm{cm}$ reduces it further to $5.9 \mathrm{eV}$. Still, (a)

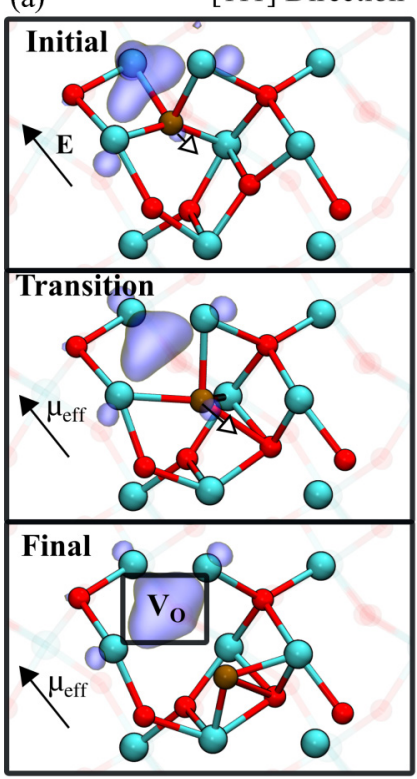

(b)

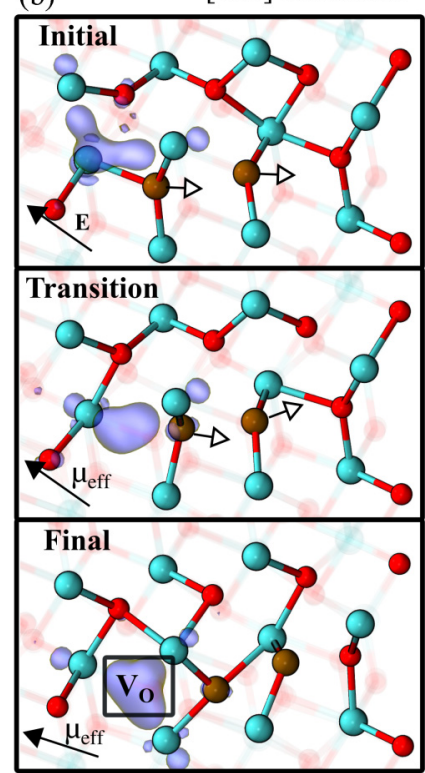

FIG. 4. Two pathways for the oxygen vacancy DP formation mechanism when a bipolaron is present. The initial, transition, and final states are shown for each of the two $\mathrm{O}$ ion displacement directions. The displaced $\mathrm{O}$ ion is in brown; the other $\mathrm{O}$ ions are in red, and $\mathrm{Hf}$ ions are in light blue. In the final state, a $V_{\mathrm{O}}^{0}+\mathrm{O}_{\mathrm{I}}^{-2}$ pair is created. Blue surfaces show the wave function of the vacancy (isovalue $=0.007$ ). (a) In the [111] direction pathway, a 4C oxygen is displaced into the $3 \mathrm{C} \mathrm{O}$ ion sublattice. (b) In the [001] direction pathway, a $3 \mathrm{C} \mathrm{O}$ ion is displaced within the $3 \mathrm{C}$ plane, displacing a neighboring $3 \mathrm{C} \mathrm{O}$ ion via an interstitialcy mechanism.

the barrier is too high to be surmounted (within a reasonable amount of time) at room temperature.

One can also estimate the barrier change using Eq. (4). The calculated dipole moment change at the transition state is $7.36 \mathrm{e} \AA$. This means that at $5.0 \mathrm{MV} / \mathrm{cm}$ and with perfect alignment, we would see a barrier reduction of $0.37 \mathrm{eV}$, and at $10.0 \mathrm{MV} / \mathrm{cm}$ there will be a reduction of $0.74 \mathrm{eV}$. These values are in good agreement with the predictions of the full DFT calculations.

\section{B. Effect of electron injection}

It was shown in previous calculations that injected electrons can form polaron and bipolaron states in $m-\mathrm{HfO}_{2}$ [42] and that the localization of electrons into intrinsic polaron states or by neutral $\mathrm{O}$ vacancies massively reduces the formation energies of oxygen DPs in $m-\mathrm{HfO}_{2}$ [14]. The initial state in Fig. 4 shows a bulk cell of $m-\mathrm{HfO}_{2}$ with two electrons trapped into a bipolaron state. In reduced $\mathrm{HfO}_{2}$ films electrons can be trapped into even deeper states by neutral $\mathrm{O}$ vacancies [14], and in amorphous films they can be trapped at low-coordinated Hf sites $[43,44]$. The localization of two electrons in $\mathrm{HfO}_{2}$ was shown to significantly reduce the barrier height for DP creation $[14,44]$. Thus, the bias application causes the electron injection, and the extra electrons reduce barriers for formation of $\mathrm{O}$ vacancies. Below we consider the effect of electric field on the barriers for defect creation and diffusion. 


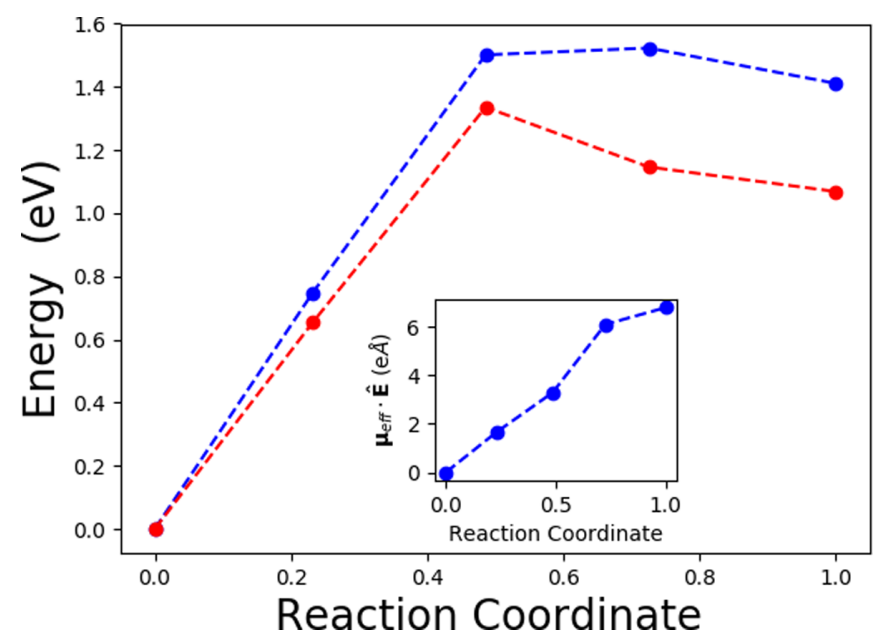

FIG. 5. The energy profile for the conversion of a bipolaron into a Frenkel pair in $m-\mathrm{HfO}_{2}$. The blue line corresponds to the process at zero field, and the red line corresponds to the process with a $5.0 \mathrm{MV} / \mathrm{cm}$ field applied in the direction of the induced dipole change. The inset shows the value of the induced dipole, projected onto the $\mathbf{E}$ field direction.

As discussed above, the $\mathbf{E}$ field will interact with charged defects in the insulator, affecting barrier heights for reactions which involve the movement of charged ions. Analyzing the possible pathways for formation of an oxygen DP from an electron bipolaron in $m$ - $\mathrm{HfO}_{2}$, we find three pathways, one of which is similar to the pathway for the neutral case discussed above. That is, the oxygen ion moves in the [100] direction as before; however, the oxygen vacancy is created in the neutral rather than positive state. This reduces the barrier height significantly to $3.3 \mathrm{eV}$.

The second pathway differs from the first and has a minimum energy barrier of approximately $1.5 \mathrm{eV}$ at zero field. The initial, transition, and final states of this process are shown in Fig. 4, and the corresponding energy profile is shown in Fig. 5. One can see that the resulting defect pair corresponds to the neutral vacancy and $\mathrm{O}_{\mathrm{I}}^{-2}$ ion. In other words, the two extra electrons are localized in the $\mathrm{O}$ vacancy. The $\mathrm{O}$ displacement corresponding to the stable nearest-neighbor DP formation is much shorter than the [100] direction pathway (as in the neutral case, for example) at about $2.53 \AA$. This is caused by the fact that the vacancy is now occupied by two electrons that repel the $\mathrm{O}_{\mathrm{I}}^{-2}$ ion which is displacing the nearest $\mathrm{O}$ ion from its site via the interstitialcy mechanism (see Fig. 4). The defect creation pathway presented in Fig. 4 is approximately along the [111] direction and involves the displacement of a $4 \mathrm{C} \mathrm{O}$ ion into the $3 \mathrm{C} \mathrm{O}$ sublattice, creating (in the final state) a $4 \mathrm{C} V_{\mathrm{O}}$ and a $3 \mathrm{C} \mathrm{O}_{\mathrm{I}}^{-2}$.

When applying an $\mathbf{E}$ field of strength $5.0 \mathrm{MV} / \mathrm{cm}$ in the direction aligned with the induced transition dipole, the maximum barrier height is reduced to $1.34 \mathrm{eV}$. Figure 5 also shows the energy profile of the reaction when modified by the presence of an $\mathbf{E}$ field. As stated above, this is similar to simply the interaction of the $\mathbf{E}$ field and the dipole induced by the defect motion. The induced dipole change is equal to $3.29 \mathrm{e} \AA$. The transition state dipole $\mu_{\text {eff }}$ for this pathway is smaller than that of the neutral pathway shown in Fig. 3. Two

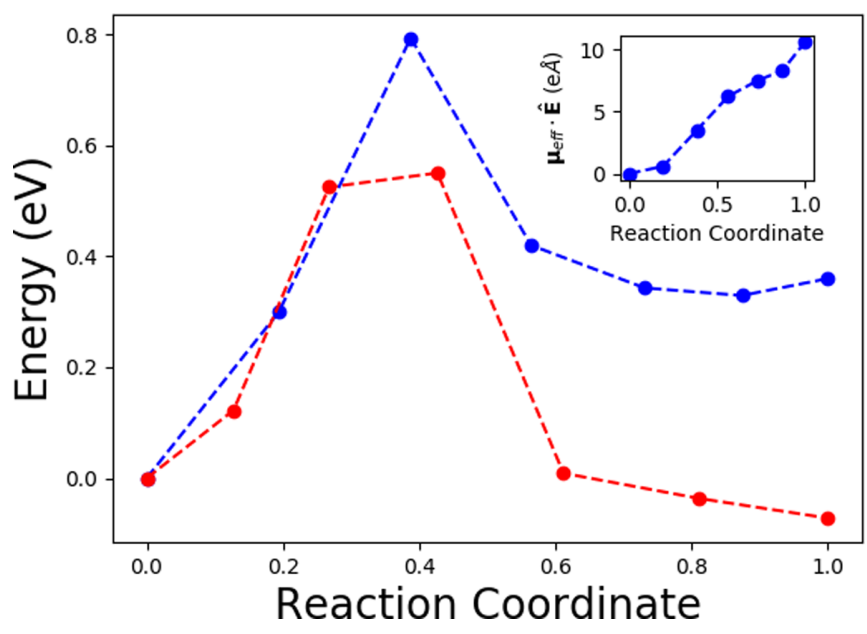

FIG. 6. The energy profile for the [001] direction pathway of DP formation shown in Fig. 4. The inset shows the value of the induced dipole, projected onto the $\mathbf{E}$ field direction.

reasons for this can be suggested. With electron injection, a $V_{\mathrm{O}}^{0}-\mathrm{O}_{\mathrm{I}}^{-2} \mathrm{DP}$ is created, whereas the neutral pathway involves generation of a $V_{\mathrm{O}}^{+2}+\mathrm{O}_{\mathrm{I}}^{-2}$ DP. For a given separation, a $(+2,-2)$ pair of charges will have a greater dipole than a $(0,-2)$ pair. Also, the defect separation in the nascent neutral DP is, in fact, larger at the transition state, further increasing $\mu_{\text {eff }}$.

In the third case, the DP is created by moving a $3 \mathrm{C} \mathrm{O}$ ion within the $3 \mathrm{C}$ sublattice and along the [001] crystal direction. In the neutral case, the creation of a DP along this direction was possible only if the defect pairs were separated by at least $7 \AA$ so that the interstitial $\mathrm{O}_{\mathrm{I}}^{-2}$ ion can be shielded by another site $\mathrm{O}$ ion from the charged vacancy. After the electron injection there is no need for a bridging $\mathrm{O}$ ion to stabilize this DP configuration as there is no Coulomb attraction between the neutral $V_{\mathrm{O}}$ and nascent $\mathrm{O}_{\mathrm{I}}^{-2}$ ion. This pathway has the lowest energy barrier of all the pathways we analyzed, at $0.82 \mathrm{eV}$ (see Fig. 6). Again, the effect of applying an $\mathbf{E}$ field was found to be closely approximated by assuming that the E field interacts with the induced dipole corresponding to the zero-field reaction.

\section{Oxygen interstitial diffusion}

The reactions described above create interstitial $\mathrm{O}_{\mathrm{I}}^{-2}$ ions. Similar to $\mathrm{O}$ ion generation, $\mathrm{O}$ diffusion may proceed along multiple directions. Only 3C-to-3C O ion diffusion is possible, however, since the $4 \mathrm{C} \mathrm{O} \mathrm{O}_{\mathrm{I}}$ defect is unstable. The $3 \mathrm{C}$ $\mathrm{O}_{\mathrm{I}}^{-2}$ defect has a split interstitial configuration: two $\mathrm{O}$ ions share the same lattice site. Here we analyze three diffusion pathways. Two of these pathways located within the $3 \mathrm{C}$ plane are in the [010] and [001] directions, as illustrated in Fig. 7. The third pathway is diffusion by the interstitialcy mechanism through the $4 \mathrm{C}$ plane (similar to the pathway discussed for the neutral DP formation) in the [100] direction.

The energy profiles for $\mathrm{O}_{\mathrm{I}}^{-2}$ diffusion along the [010] and [001] directions are shown in Fig. 8. Again, energy profiles calculated using both the periodic $\mathbf{E}$ field and dipoleinteraction method are approximately equal. However, the 
(a)

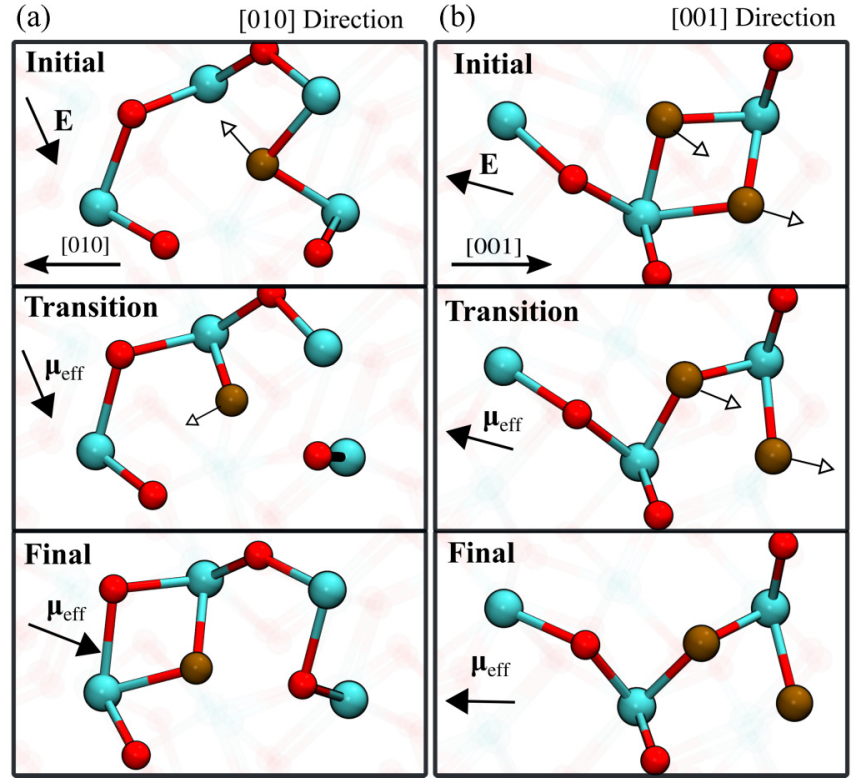

FIG. 7. Interstitial diffusion pathways within the $3 \mathrm{C} \mathrm{O}$ plane. Both directions within the plane (both the [010] direction and the [001] direction) are possible and have similar activation energies.

$\mathrm{O}_{\mathrm{I}}^{-2}$ diffusion along the [010] direction has a bent trajectory with the dipole moment at initial and final states perpendicular to the field direction. This alignment at one point of the trajectory favors the formation of a new energy minimum, as can be seen in Fig. 9.

For either pathway, the interstitial oxygen defect has a low activation energy (approximately $0.1 \mathrm{eV}$ ) once a strong field of $10 \mathrm{MV} / \mathrm{cm}$ is applied. This means that, once a DP is created, there should be rapid separation of the defect species, as expected for a breakdown model. Diffusion in the [100] direction via substitution of a 4C O ion has a higher energy barrier at $1.9 \mathrm{eV}$. This suggests that, in a perfect $m-\mathrm{HfO}_{2}$ lattice, $\mathrm{O}_{\mathrm{I}}^{-2}$ diffusion should be mostly confined to within a $3 \mathrm{C} \mathrm{O}$ plane where the DP formation energy is also the

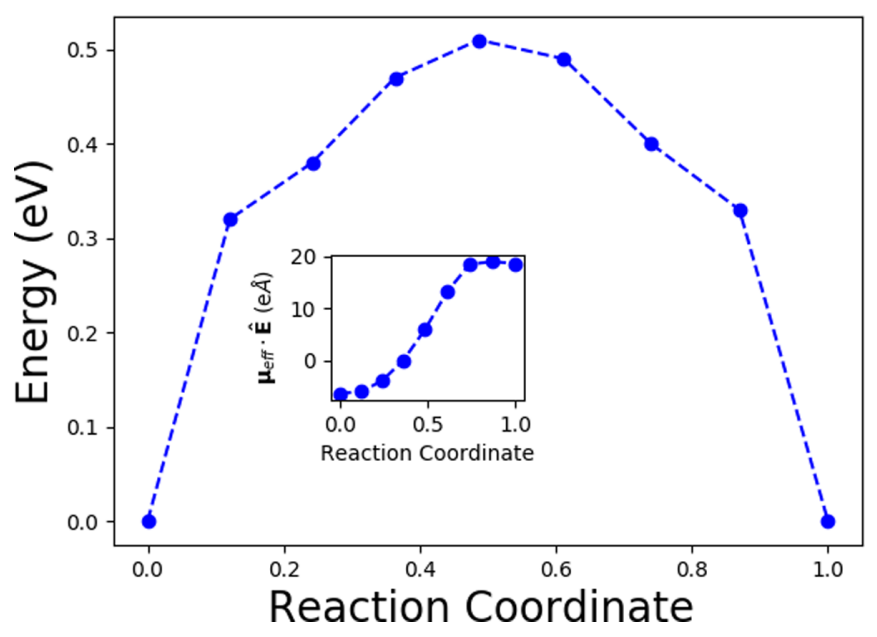

FIG. 8. Energy profile of the [001] oxygen interstitial diffusion mechanism in $m-\mathrm{HfO}_{2}$. The blue line refers to the zero-field scenario. The inset shows $\mu_{\text {eff }}$ in the direction parallel to the $\mathbf{E}$ field, which gives the maximum barrier reduction.

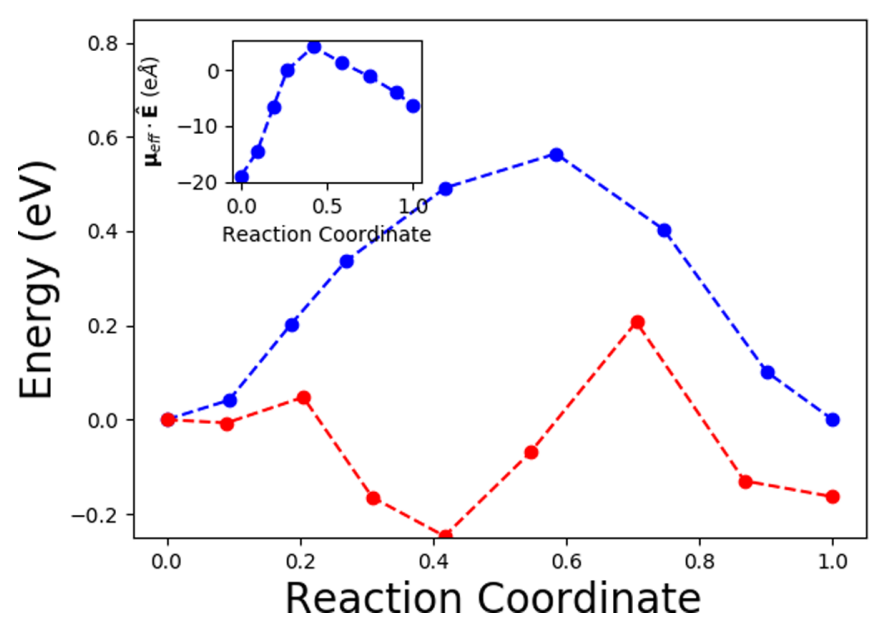

FIG. 9. Energy profile for the pathway shown in Fig. 7(b). The blue line corresponds to the zero-field energy pathway, whereas the red line is the profile under field application. The reaction coordinate is defined for each plot, in the same way as for other energy profiles.

lowest. We note that the anisotropic diffusion of $\mathrm{O}$ interstitials via an interstitialcy mechanism was observed in simulations of, e.g., the perovskite-related materials, the RuddlesdenPopper series of tetragonal layered oxides [45,46]. Experimental observation of such processes is challenging, especially in polycrystalline films, such as $\mathrm{HfO}_{2}$. However, such an anisotropic oxygen diffusion was recently visualized in $\mathrm{Ce}_{2} \mathrm{O}_{3}$ using high-resolution Transmission electron microscopy and simulations [47].

\section{CONCLUSION}

We have investigated the effect of the $\mathbf{E}$ field on energy barriers for defect generation and $\mathrm{O}_{\mathrm{I}}$ diffusion in $m-\mathrm{HfO}_{2}$ for field strengths up to $10 \mathrm{MV} / \mathrm{cm}$. By direct calculation of each effect, we demonstrated that electron injection into the oxide and localization on defect or trap states are the main cause of the energy barrier reduction in the field-induced generation of oxygen vacancies. This is in disagreement with other models, such as the thermochemical model, suggesting that the main effect is, rather, a reduction to the activation energy caused by direct interaction between the electric field and bond dipoles present in the pristine system. Using direct calculation of the reaction pathway under an applied $\mathbf{E}$ field, we demonstrated that the field-induced reduction to the activation energy does not exceed $1 \mathrm{eV}$ even at breakdown voltage and is much smaller than the reduction caused by trapped electrons. Our results confirm that even at fields approaching $10 \mathrm{MV} / \mathrm{cm}$ the barrier height change for the defect creation can be well approximated by calculating the transition state dipole moment in the zero-field system. Thus, to fully understand the degradation process, the complex interplay between the effects of the external field, electron injection and localization, defect creation, and ion diffusion must be considered. We do not expect the energetics to differ greatly for other phases of $\mathrm{HfO}_{2}$. For example, previous calculations of oxygen vacancies in amorphous $\mathrm{HfO}_{2}$ [48] showed formation energies very similar to oxygen vacancies in $m-\mathrm{HfO}_{2}$. 
The obtained reaction and diffusion barriers and their dependence on the strength of the electric field will later be used in simulations of degradation and dielectric breakdown of $\mathrm{HfO}_{2}$ films.

\section{ACKNOWLEDGMENTS}

We acknowledge funding provided by the UK Engineering and Physical Sciences Research Council (EPSRC) under
Grants No. EP/K01739X/1 and No. EP/P013503/1 and by the Leverhulme Trust RPG-2016-135. Computer facilities on the ARCHER UK National Supercomputing Service have been provided via the United Kingdom's HPC Materials Chemistry Consortium (EPSRC Grant No. EP/L000202). We would like to thank A.-M. El-Sayed and A. Padovani for useful discussions and sharing the results of their work.
[1] G Groeseneken, R. Degraeve, T. Nigam, G. Van den bosch, and H. E. Maes, Hot carrier degradation and time-dependent dielectric breakdown in oxides, Microelectron. Eng. 49, 27 (1999).

[2] J. Verweij and J. Klootwijk, Dielectric breakdown I: A review of oxide breakdown, Microelectron. J. 27, 611 (1996).

[3] D. DiMaria, Defect production, degradation, and breakdown of silicon dioxide films, Solid-State Electron. 41, 957 (1997).

[4] R. Degraeve, B. Kaczer, and G. Groeseneken, Degradation and breakdown in thin oxide layers: Mechanisms, models and reliability prediction, Microelectron. Reliab. 39, 1445 (1999).

[5] S. Lombardo, J. H. Stathis, B. P. Linder, K. L. Pey, F. Palumbo, and C. H. Tung, Dielectric breakdown mechanisms in gate oxides, J. Appl. Phys. 98, 121301 (2005).

[6] N. Raghavan, K. L. Pey, and K. Shubhakar, High-k dielectric breakdown in nanoscale logic devices scientific insight and technology impact, Microelectron. Reliab. 54, 847 (2014).

[7] J. W. McPherson and H. C. Mogul, Underlying physics of the thermochemical $\mathrm{E}$ model in describing low-field timedependent dielectric breakdown in $\mathrm{SiO}_{2}$ thin films, J. Appl. Phys. 84, 1513 (1998).

[8] J. McPherson, J.-Y. Kim, A. Shanware, and H. Mogul, Thermochemical description of dielectric breakdown in high dielectric constant materials, Appl. Phys. Lett. 82, 2121 (2003).

[9] I.-C. Chen, S. Holland, and C. Hu, Electrical breakdown in thin gate and tunneling oxides, IEEE Trans. Electron Devices 32, 413 (1985).

[10] M. S. Akbar, C. H. Choi, S. J. Rhee, S. A. Krishnan, C. Y. Kang, M. H. Zhang, T. Lee, I. J. Ok, F. Zhu, H.-S. Kim, and J. C. Lee, A novel approach in separating the roles of electrons and holes in causing degradation in Hf-based MOSFET devices by using stress-anneal technique, IEEE Electron Device Lett. 28, 132 (2007).

[11] E. Cartier and A. Kerber, Stress-induced leakage current and defect generation in nFETs with $\mathrm{HfO}_{2} / \mathrm{TiN}$ gate stacks during positive-bias temperature stress, in IEEE 47th Annual International Reliability Physics Symposium (IEEE, Piscataway, NJ, 2009), pp. 486-492.

[12] K. Okada, M. Kamei, and S. Ohno, Reconsideration of dielectric breakdown mechanism of gate dielectrics on basis of dominant carrier change model, IEEE Trans. Electron Devices 64, 4386 (2017).

[13] B. Traore, P. Blaise, and B. Sklenard, Reduction of monoclinic $\mathrm{HfO}_{2}$ : A cascading migration of oxygen and its interplay with a high electric field, J. Phys. Chem. C 120, 25023 (2016).
[14] S. R. Bradley, A. L. Shluger, and G. Bersuker, Electron-Injection-Assisted Generation of Oxygen Vacancies in Monoclinic $\mathrm{HfO}_{2}$, Phys. Rev. Appl. 4, 064008 (2015).

[15] J. Strand, M. Kaviani, and A. L. Shluger, Defect creation in amorphous $\mathrm{HfO}_{2}$ facilitated by hole and electron injection, Microelectron. Eng. 178, 279 (2017).

[16] A. Padovani, D. Gao, A. Shluger, and L. Larcher, A microscopic mechanism of dielectric breakdown in $\mathrm{SiO}_{2}$ films: An insight from multi-scale modeling, J. Appl. Phys. 121, 155101 (2017).

[17] MDLsoft Ginestra ${ }^{T M}$, https://mdlsoft.com/, Manual - Physics of Carrier Transport in Ginestra (2018).

[18] N. Cabrera and N. F. Mott, Theory of the oxidation of metals, Rep. Prog. Phys. 12, 163 (1949).

[19] A. T. Fromhold and E. L. Cook, Diffusion currents in large electric fields for discrete lattices, J. Appl. Phys. 38, 1546 (1967).

[20] L. Vandelli, A. Padovani, L. Larcher, and G. Bersuker, Microscopic modeling of electrical stress-induced breakdown in polycrystalline hafnium oxide dielectrics, IEEE Trans. Electron Devices 60, 1754 (2013).

[21] R. D. King-Smith and D. Vanderbilt, Theory of polarization of crystalline solids, Phys. Rev. B 47, 1651 (1993).

[22] R. Resta, Macroscopic polarization in crystalline dielectrics: The geometric phase approach, Rev. Mod. Phys. 66, 899 (1994).

[23] I. Souza, J. Iñiguez, and D. Vanderbilt, First-Principles Approach to Insulators in Finite Electric Fields, Phys. Rev. Lett. 89, 117602 (2002).

[24] P. Umari and A. Pasquarello, Ab Initio Molecular Dynamics in a Finite Homogeneous Electric Field, Phys. Rev. Lett. 89, 157602 (2002).

[25] N. A. Spaldin, A beginners guide to the modern theory of polarization, J. Solid State Chem. 195, 2 (2012).

[26] A.-M. El-Sayed, M. B. Watkins, T. Grasser, and A. L. Shluger, Effect of electric field on migration of defects in oxides: Vacancies and interstitials in bulk MgO, Phys. Rev. B 98, 064102 (2018).

[27] G. Rzepa, J. Franco, B. O’Sullivan, A. Subirats, M. Simicic, G. Hellings, P. Weck, M. Jech, T. Knobloch, M. Waltl, P. J. Roussel, D. Linten, B. Kaczer, and T. Grasser, ComphyA compact-physics framework for unified modeling of BTI, Microelectron. Reliab. 85, 49 (2018).

[28] J. Lippert, G. Hutter, and M. Parrinelo, A hybrid Gaussian and plane wave density functional scheme, Mol. Phys. 92, 477 (1997). 
[29] J. VandeVondele, M. Krack, F. Mohamed, M. Parrinello, T. Chassaing, and J. Hutter, Quickstep: Fast and accurate density functional calculations using a mixed Gaussian and plane waves approach, Comput. Phys. Commun. 167, 103 (2005).

[30] M. Guidon, J. Hutter, and J. VandeVondele, Robust periodic Hartree-Fock exchange for large-scale simulations using Gaussian basis sets, J. Chem. Theory Comput. 5, 3010 (2009).

[31] M. Guidon, J. Hutter, and J. VandeVondele, Auxiliary density matrix methods for Hartree- Hock exchange calculations, J. Chem. Theory Comput. 6, 2348 (2010).

[32] J. VandeVondele and J. Hutter, Gaussian basis sets for accurate calculations on molecular systems in gas and condensed phases, J. Chem. Phys. 127, 114105 (2007).

[33] S. Goedecker, M. Teter, and J. Hutter, Separable dual-space Gaussian pseudopotentials, Phys. Rev. B 54, 1703 (1996).

[34] K. P. McKenna, M. J. Wolf, A. L. Shluger, S. Lany, and A. Zunger, Two-Dimensional Polaronic Behavior in the Binary Oxides $m-\mathrm{HfO}_{2}$ and $m-\mathrm{ZrO}_{2}$, Phys. Rev. Lett. 108, 116403 (2012).

[35] K. Xiong and J. Robertson, Point defects in $\mathrm{HfO}_{2}$ high k gate oxide, Microelectron. Eng. 80, 408 (2005).

[36] J. Gavartin, D. Muñoz Ramo, A. Shluger, G. Bersuker, and B. Lee, Negative oxygen vacancies in $\mathrm{HfO}_{2}$ as charge traps in highk stacks, Appl. Phys. Lett. 89, 082908 (2006).

[37] R. Öttking, S. Kupke, E. Nadimi, R. Leitsmann, F. Lazarevic, P. Plänitz, G. Roll, S. Slesazeck, M. Trentzsch, and T. Mikolajick, Defect generation and activation processes in $\mathrm{HfO}_{2}$ thin films: Contributions to stress-induced leakage currents, Phys. Status Solidi A 212, 547 (2015).

[38] A. S. Foster, A. L. Shluger, and R. M. Nieminen, Mechanism of Interstitial Oxygen Diffusion in Hafnia, Phys. Rev. Lett. 89, 225901 (2002).
[39] R. Elber and M. Karplus, A method for determining reaction paths in large molecules: Application to myoglobin, Chem. Phys. Lett. 139, 375 (1987).

[40] G. Henkelman, B. P. Uberuaga, and H. Jónsson, A climbing image nudged elastic band method for finding saddle points and minimum energy paths, J. Chem. Phys. 113, 9901 (2000).

[41] G. Henkelman and H. Jónsson, Improved tangent estimate in the nudged elastic band method for finding minimum energy paths and saddle points, J. Chem. Phys. 113, 9978 (2000).

[42] D. M. Ramo, J. Gavartin, A. Shluger, and G. Bersuker, Intrinsic and defect-assisted trapping of electrons and holes in $\mathrm{HfO}_{2}$ : An ab initio study, Microelectron. Eng. 84, 2362 (2007).

[43] M. Kaviani, J. Strand, V. V. Afanas' ev, and A. L. Shluger, Deep electron and hole polarons and bipolarons in amorphous oxide, Phys. Rev. B 94, 020103(R) (2016).

[44] J. Strand, M. Kaviani, V. V. Afanasev, J. G. Lisoni, and A. L. Shluger, Intrinsic electron trapping in amorphous oxide, Nanotechnology 29, 125703 (2018).

[45] A. Chroneos, D. Parfitt, J. A. Kilner, and R. W. Grimes, Anisotropic oxygen diffusion in tetragonal $\mathrm{La}_{2} \mathrm{NiO}_{4}+\mathrm{d}$ : molecular dynamics calculations, J. Mater. Chem. 20, 266 (2010).

[46] A. Kushima, D. Parfitt, A. Chroneos, B. Yildiz, J. A. Kilner, and R. W. Grimes, Interstitialcy diffusion of oxygen in tetragonal $\mathrm{La}_{2} \mathrm{CoO}_{4}+\mathrm{d}$, Phys. Chem. Chem. Phys. 13, 2242 (2011).

[47] L. Zhu, X. Jin, Y.-Y. Zhang, S. Du, L. Liu, T. Rajh, Z. Xu, W. Wang, X. Bai, J. Wen, and L. Wang, Visualizing Anisotropic Oxygen Diffusion in Ceria Under Activated Conditions, Phys. Rev. Lett. 124, 056002 (2020).

[48] F. Cerbu, O. Madia, D. Andreev, S. Fadida, M. Eizenberg, L. Breuil, J. Lisoni, J. Kittl, J. Strand, A. Shluger et al., Intrinsic electron traps in atomic-layer deposited $\mathrm{HfO}_{2}$ insulators, Appl. Phys. Lett. 108, 222901 (2016). 